\title{
Some Fixed Point Theorems in Generalized Probabilistic Metric Spaces
}

\author{
Chuanxi Zhu, Wenqing Xu, and Zhaoqi Wu \\ Department of Mathematics, Nanchang University, Nanchang 330031, China \\ Correspondence should be addressed to Chuanxi Zhu; chuanxizhu@126.com \\ Received 4 July 2014; Accepted 16 September 2014; Published 16 October 2014 \\ Academic Editor: Simeon Reich
}

Copyright (c) 2014 Chuanxi Zhu et al. This is an open access article distributed under the Creative Commons Attribution License, which permits unrestricted use, distribution, and reproduction in any medium, provided the original work is properly cited.

\begin{abstract}
We introduce the concepts of $(H, \psi, \Phi)$-contraction and probabilistic $(\alpha, \varphi)$-contraction mappings in generalized probabilistic metric spaces and prove some fixed point theorems for such two types of mappings in generalized probabilistic metric spaces. Our results generalize and extend many comparable results in existing literature. Some examples are also given to support our results. Finally, an application to the existence of solutions for a class of integral equations is presented by utilizing one of our main results.
\end{abstract}

\section{Introduction and Preliminaries}

The notion of a probabilistic metric space was introduced and studied by Menger [1]. The idea of Menger was to use distribution functions instead of nonnegative real numbers to describe the distance between two points. It has become an active field since then and many fixed point results for mappings satisfying different contractive conditions have been studied [2-8].

On the other hand, Mustafa and Sims [9] defined the concept of a $G$-metric space and presented the Banach fixed point theorem in the context of a complete $G$-metric space. Following their results, some authors have obtained many fixed point theorems for contractive mappings in $G$-metric spaces [9-16].

As a generalization, using PM-spaces and G-metric spaces, Zhou et al. [17] defined the notion of generalized probabilistic metric spaces (or probabilistic $G$-metric spaces). The purpose of this paper is to establish some fixed point theorems for two types of mappings satisfying the $(H, \psi, \Phi)$ contraction or probabilistic $(\alpha, \varphi)$-contraction in generalized probabilistic metric spaces. As consequences, our results generalize and extend many comparable results (see, e.g., [6$8,14,16,17])$.

We introduce some useful concepts and lemmas for the development of our results.
Let $R$ denote the set of reals and $R^{+}$the nonnegative reals. A mapping $F: R \rightarrow R^{+}$is called a distribution function if it is nondecreasing and left continuous with $\inf _{t \in R} F(t)=0$ and $\sup _{t \in R} F(t)=1$. We will denote by $D$ the set of all distribution functions and let $D^{+}=\{F \in D: F(t)=0, \forall t \leq 0\}$.

Let $H$ denote the specific distribution function defined by

$$
H(t)= \begin{cases}0, & t \leq 0 \\ 1, & t>0\end{cases}
$$

Definition 1 (see [2]). The mapping $\Delta:[0,1] \times[0,1] \rightarrow$ $[0,1]$ is called a triangular norm (for short, a $t$-norm) if the following conditions are satisfied:

$$
\begin{aligned}
& (\Delta-1) \Delta(a, 1)=a, \text { for all } a \in[0,1] \\
& (\Delta-2) \Delta(a, b)=\Delta(b, a) ; \\
& (\Delta-3) \Delta(a, b) \leq \Delta(c, d), \text { for } c \geq a, d \geq b ; \\
& (\Delta-4) \Delta(a, \Delta(b, c))=\Delta(\Delta(a, b), c) .
\end{aligned}
$$

Three sample examples of continuous $t$-norms are $\Delta_{1}(a, b)=\max \{a+b-1,0\}, \Delta_{2}(a, b)=a b$, and $\Delta_{M}(a, b)=$ $\min \{a, b\}$ for all $a, b \in[0,1]$.

Definition 2 (see [17]). A Menger probabilistic G-metric space (briefly, a Menger PGM-space) is a triplet $\left(X, G^{*}, \Delta\right)$, 
where $X$ is a nonempty set, $\Delta$ is a continuous $t$-norm, and $G^{*}$ is a mapping from $X \times X \times X$ into $D^{+}\left(G_{x, y, z}^{*}\right.$ denotes the value of $G^{*}$ at the point $\left.(x, y, z)\right)$ satisfying the following conditions:

$(\mathrm{PGM}-1) G_{x, y, z}^{*}(t)=1$ for all $x, y, z \in X$ and $t>0$ if and only if $x=y=z$;

$(\mathrm{PGM}-2) G_{x, x, y}^{*}(t) \geq G_{x, y, z}^{*}(t)$ for all $x, y \in X$ with $z \neq y$ and $t>0$;

$(\mathrm{PGM}-3) G_{x, y, z}^{*}(t)=G_{y, x, z}^{*}(t)=G_{y, z, x}^{*}(t)=\cdots$ (symmetry in all three variables);

$(\mathrm{PGM}-4) G_{x, y, z}^{*}(t+s) \geq \Delta\left(G_{x, a, a}^{*}(t), G_{a, y, z}^{*}(s)\right)$ for all $x, y, z, a \in X$ and $t, s \geq 0$.

Example 3. Let $(X, d)$ be an ordinary metric space. Define $G_{s}^{*}(x, y, z)(t)=H(t-k(d(x, y)+d(y, z)+d(x, z)))$ and $G_{m}^{*}(x, y, z)(t)=H(t-\max \{d(x, y), d(y, z), d(x, z)\})$, where $k>0$. Then $\left(X, G_{s}^{*}, \Delta_{M}\right)$ and $\left(X, G_{m}^{*}, \Delta_{M}\right)$ are both Menger PGM-spaces.

Example 4. Let $Q$ be a distribution function, $Q(0)=0$, and

$$
G_{x, y, z}^{*}(t)= \begin{cases}Q(t), & \text { at least two of } x, y, z \text { are distinct } \\ H(t), & x=y=z\end{cases}
$$

Then $\left(X, G^{*}, \Delta_{M}\right)$ is a Menger PGM-space.

Example 5. Let $(X, d)$ be an ordinary metric space, let $Q$ be a distribution function different from $H$, satisfying $Q(0)=0$, and

$$
G_{x, y, z}^{*}(t)=\left\{\begin{array}{l}
Q\left(\frac{t}{d(x, y)+d(y, z)+d(x, z)}\right) \\
\text { at least two of } x, y, z \text { are distinct, } \\
H(t), \quad x=y=z .
\end{array}\right.
$$

Then $\left(X, G^{*}, \Delta_{M}\right)$ is a Menger PGM-space.

Remark 6 (see [17]). Zhou et al. pointed out that if $\left(X, G^{*}, \Delta\right)$ is a Menger PGM-space and $\Delta$ is continuous, then $\left(X, G^{*}, \Delta\right)$ is a Hausdorff topological space in the $(\varepsilon, \lambda)$-topology $T$; that is, the family of sets $\left\{N_{x}(\varepsilon, \lambda): \varepsilon>0, \lambda \in(0,1]\right\}(x \in X)$ is a basis of neighborhoods of a point $x$ for $T$, where $N_{x}(\varepsilon, \lambda)=$ $\left\{y \in X: G_{x, y, y}^{*}(\varepsilon)>1-\lambda, G_{y, x, x}^{*}(\varepsilon)>1-\lambda\right\}$.

Definition 7 (see [17]). Let $\left(X, G^{*}, \Delta\right)$ be a PGM-space.

(1) A sequence $\left\{x_{n}\right\}$ in $X$ is said to be convergent to a point $x$ in $X$ (write $x_{n} \rightarrow x$ ) if, for any $\varepsilon>0$ and $0<\lambda<1$, there exists a positive integer $M_{\varepsilon, \lambda}$ such that $x_{n} \in N_{x}(\varepsilon, \lambda)$, whenever $n>M_{\varepsilon, \lambda}$.

(2) A sequence $\left\{x_{n}\right\}$ in $X$ is called a Cauchy sequence if, for any $\varepsilon>0$ and $0<\lambda<1$, there exists a positive integer $M_{\varepsilon, \lambda}$, such that $G_{x_{n}, x_{m}, x_{l}}^{*}(\varepsilon)>1-\lambda$, whenever $n, m, l>M_{\varepsilon, \lambda}$.
(3) A PGM-space $\left(X, G^{*}, \Delta\right)$ is said to be complete if every Cauchy sequence in $X$ converges to a point in $X$.

(4) A mapping $T: X \rightarrow X$ is said to be continuous at a point $x \in X$ if $\left\{x_{n}\right\}$ is convergent to $x$ implying that $\left\{T x_{n}\right\}$ is convergent to $T x$.

Definition 8 (see [3]). A $t$-norm $\Delta$ is said to be of $H$-type if the family of functions $\left\{\Delta^{m}(t)\right\}_{m=1}^{\infty}$ is equicontinuous at $t$, where $\Delta^{1}(t)=\Delta(t, t)$ and $\Delta^{m}(t)=\Delta\left(t, \Delta^{m-1}(t)\right), m=1,2, \ldots, t \in$ $[0,1]$.

Definition 9 (see [4]). A function $\Phi: R^{+} \rightarrow R^{+}$is said to satisfy the condition $(\Phi)$ if it is strictly increasing, rightcontinuous and $\Phi^{n}(t) \rightarrow 0, t>0$, as $n \rightarrow \infty$, where $\Phi^{n}(t)$ is the $n$th iteration of $\Phi(t)$.

Lemma 10 (see [6]). Suppose that $F \in D^{+}$. For every $n \in Z^{+}$, let $F_{n}: R \rightarrow[0,1]$ be nondecreasing and $g_{n}:(0,+\infty) \rightarrow$ $(0,+\infty)$ satisfy $\lim _{n \rightarrow \infty} g_{n}(t)=1$ for any $t>0$. If $F_{n}\left(g_{n}(t)\right) \geq$ $F(t)$ for any $t>0$, then $\lim _{n \rightarrow \infty} F_{n}(t)=1$ for any $t>0$.

Lemma 11 (see [17]). Let $\left(X, G^{*}, \Delta\right)$ be a Menger PGM-space. Let $\left\{x_{n}\right\},\left\{y_{n}\right\}$, and $\left\{z_{n}\right\}$ be sequences in $X$ and $x, y, z \in X$. If $x_{n} \rightarrow x, y_{n} \rightarrow y$, and $z_{n} \rightarrow z$ as $n \rightarrow \infty$, then, for any $t>0, G_{x_{n}, y_{n}, z_{n}}^{*}(t) \rightarrow G_{x, y, z}^{*}(t)$ as $n \rightarrow \infty$.

\section{Main Results}

In this section, we will give some fixed point theorems for two types of mappings satisfying the $(H, \psi, \Phi)$-contraction or probabilistic $(\alpha, \varphi)$-contraction in generalized probabilistic metric spaces. We first present some useful lemmas.

Lemma 12. Let $\left(X, G^{*}, \Delta\right)$ be a Menger PGM-space and $\Delta$ be a continuous t-norm. Then the following statements are equivalent:

(i) the sequence $\left\{x_{n}\right\}$ is a Cauchy sequence;

(ii) for any $\varepsilon>0$ and $0<\lambda<1$, there exists $M \in N^{+}$such that $G_{x_{n}, x_{m}, x_{m}}^{*}(\varepsilon)>1-\lambda$, for all $n, m>M$.

Proof. (i) $\Rightarrow$ (ii). This can be easily obtained from Definition 7 (2).

(ii) $\Rightarrow$ (i). Since $\Delta$ is continuous, for every $\varepsilon>0$ and $\lambda>0$, there exists $\lambda_{0} \in(0, \lambda)$, such that $\Delta\left(1-\lambda_{0}, 1-\lambda / 2\right)>1-\lambda$. Let $\lambda_{1}=\min \left\{\lambda_{0}, \lambda / 2\right\}$. Then $\Delta\left(1-\lambda_{1}, 1-\lambda_{1}\right)>1-\lambda$. Hence, from (ii), there exists $M \in N^{+}$, such that $G_{x_{n}, x_{m}, x_{m}}^{*}(\varepsilon / 2)>1-\lambda_{1}$ and $G_{x_{l}, x_{m}, x_{m}}^{*}(\varepsilon / 2)>1-\lambda_{1}$, for all $n, m, l \geq M$. Then we have $G_{x_{n}, x_{m}, x_{l}}^{*}(\varepsilon) \geq \Delta\left(G_{x_{n}, x_{m}, x_{m}}^{*}(\varepsilon / 2), G_{x_{l}, x_{m}, x_{m}}^{*}(\varepsilon / 2)\right) \geq \Delta\left(1-\lambda_{1}, 1-\right.$ $\left.\lambda_{1}\right)>1-\lambda$. Thus, $\left\{x_{n}\right\}$ is a Cauchy sequence.

Lemma 13. Let $\left(X, G^{*}, \Delta\right)$ be a Menger PGM-space. For each $\lambda \in(0,1]$, define a function $G_{\lambda}^{*}$ by

$$
G_{\lambda}^{*}(x, y, z)=\inf _{t}\left\{t \geq 0: G_{x, y, z}^{*}(t)>1-\lambda\right\},
$$

for $x, y, z \in X$. Then 
(1) $G_{\lambda}^{*}(x, y, z)<t$ if and only if $G_{x, y, z}^{*}(t)>1-\lambda$;

(2) $G_{\lambda}^{*}(x, y, z)=0$ for all $\lambda \in(0,1]$ if and only if $x=y=$ $z$;

(3) $G_{\lambda}^{*}(x, y, z)=G_{\lambda}^{*}(y, x, z)=G_{\lambda}^{*}(y, z, x)=\cdots$;

(4) if $\Delta=\Delta_{M}$, then, for every $\lambda \in(0,1], G_{\lambda}^{*}(x, y, z) \leq$ $G_{\lambda}^{*}(x, a, a)+G_{\lambda}^{*}(a, y, z)$.

Proof. It is not difficult to prove that (1), (2), and (3) hold. Now, we prove that (4) also holds. For any $\lambda \in(0,1]$ and $\varepsilon>0$, we have $G_{x, a, a}^{*}\left(G_{\lambda}^{*}(x, a, a)+\varepsilon / 2\right)>1-\lambda$ and $G_{a, y, z}^{*}\left(G_{\lambda}^{*}(a, y, z)+\varepsilon / 2\right)>1-\lambda$. Hence, from (PGM - 4) and $\Delta=\Delta_{M}$, we have

$$
\begin{gathered}
G_{x, y, z}^{*}\left(G_{\lambda}^{*}(x, a, a)+G_{\lambda}^{*}(a, y, z)+\varepsilon\right) \\
\geq \min \left\{G_{x, a, a}^{*}\left(G_{\lambda}^{*}(x, a, a)+\frac{\varepsilon}{2}\right),\right. \\
\left.G_{a, y, z}^{*}\left(G_{\lambda}^{*}(a, y, z)+\frac{\varepsilon}{2}\right)\right\} \\
>\min \{1-\lambda, 1-\lambda\}=1-\lambda,
\end{gathered}
$$

which implies that $G_{\lambda}^{*}(x, y, z) \leq G_{\lambda}^{*}(x, a, a)+G_{\lambda}^{*}(a, y, z)+\varepsilon$. Letting $\varepsilon \rightarrow 0$, we have $G_{\lambda}^{*}(x, y, z) \leq G_{\lambda}^{*}(x, a, a)+G_{\lambda}^{*}(a, y, z)$ for all $x, y, z, a \in X$.

Lemma 14. Let $\left(X, G^{*}, \Delta\right)$ be a Menger PGM-space and let $\left\{G_{\lambda}^{*}\right\}_{\lambda \in(0,1]}$ be a family of functions on $X$ defined by (4). If $\Delta$ is a $t$-norm of $H$-type, then, for each $\lambda \in(0,1]$, there exists $\mu \in(0, \lambda]$, such that, for each $m \in Z^{+}$,

$$
\begin{aligned}
G_{\lambda}^{*}\left(x_{0}, x_{m}, x_{m}\right) & \leq \sum_{i=0}^{m-1} G_{\mu}^{*}\left(x_{i}, x_{i+1}, x_{i+1}\right), \\
G_{\lambda}^{*}\left(x_{0}, x_{0}, x_{m}\right) & \leq \sum_{i=0}^{m-1} G_{\mu}^{*}\left(x_{i}, x_{i}, x_{i+1}\right),
\end{aligned}
$$

for all $x_{0}, x_{1}, \ldots, x_{m} \in X$.

Proof. Since $\Delta$ is a $t$-norm of $H$-type, there exists $\mu \in(0, \lambda]$, such that, for all $m \in Z^{+}$,

$$
\Delta^{m}(1-\mu)>1-\lambda
$$

For any given $m \in Z^{+}$and $x_{0}, x_{1}, \ldots, x_{m} \in X$, we put $G_{\mu}^{*}\left(x_{i}, x_{i+1}, x_{i+1}\right)=t_{i}(i=0,1,2, \ldots, m-1)$. For every $\varepsilon>0$, it is evident that $G_{\mu}^{*}\left(x_{i}, x_{i+1}, x_{i+1}\right)<t_{i}+\varepsilon / m$. By Lemma 13, for $i=0,1,2, \ldots, m-1$, we have

$$
G_{x_{i}, x_{i+1}, x_{i+1}}^{*}\left(t_{i}+\frac{\varepsilon}{m}\right)>1-\mu .
$$

By (7) and (8), we have

$$
\begin{gathered}
G_{x_{0}, x_{m}, x_{m}}^{*}\left(t_{0}+t_{1}+\cdots+t_{m-1}+\varepsilon\right) \\
\geq \Delta\left(G_{x_{0}, x_{1}, x_{1}}^{*}\left(t_{0}+\frac{\varepsilon}{m}\right),\right. \\
\Delta\left(G_{x_{1}, x_{2}, x_{2}}^{*}\left(t_{1}+\frac{\varepsilon}{m}\right),\right.
\end{gathered}
$$

$$
\begin{gathered}
\Delta\left(\ldots, \Delta\left(G_{x_{m-2}, x_{m-1}, x_{m-1}}^{*}\left(t_{m-2}+\frac{\varepsilon}{m}\right),\right.\right. \\
\left.\left.\left.\left.G_{x_{m-1}, x_{m}, x_{m}}^{*}\left(t_{m-1}+\frac{\varepsilon}{m}\right)\right) \cdots\right)\right)\right) \\
\geq \Delta^{m}(1-\mu)>1-\lambda .
\end{gathered}
$$

Again by Lemma 13, we get $G_{\lambda}^{*}\left(x_{0}, x_{m}, x_{m}\right)<t_{0}+t_{1}+\cdots+$ $t_{m-1}+\varepsilon$. Letting $\varepsilon \rightarrow 0$, for all $x_{0}, x_{1}, \ldots, x_{m} \in X$, we have $G_{\lambda}^{*}\left(x_{0}, x_{m}, x_{m}\right) \leq \sum_{i=0}^{m-1} G_{\mu}^{*}\left(x_{i}, x_{i+1}, x_{i+1}\right)$. Similarly, we have $G_{\lambda}^{*}\left(x_{0}, x_{0}, x_{m}\right) \leq \sum_{i=0}^{m-1} G_{\mu}^{*}\left(x_{i}, x_{i}, x_{i+1}\right)$.

Inspired by the notion of $H$-contraction mappings in [8], we introduce the notion of $(H, \psi, \Phi)$-contraction mappings in PGM-spaces.

Definition 15. Let $\left(X, G^{*}, \Delta\right)$ be a Menger PGM-space, $\Phi$ satisfy the condition $(\Phi)$ and $\psi:(-\infty, 1) \rightarrow(-\infty, 1)$ be a nondecreasing function, $\psi(s)=0$ if and only if $s=0$, $\lim _{s \rightarrow 1^{-}} \psi(s)=1$. A mapping $T: X \rightarrow X$ is said to be a $(H, \psi, \Phi)$-contraction mapping on $\left(X, G^{*}\right)$, if, for any $t>0$ and $x, y, z \in X$,

$$
G_{T x, T y, T z}^{*}(\Phi(t))>\psi(1-\Phi(t)),
$$

whenever $G_{x, y, z}^{*}(t)>\psi(1-t)$. If $\psi(s)=s$ for all $s \in(-\infty, 1)$, then $T$ is said to be a $(H, \Phi)$-contraction mapping.

Theorem 16. Let $\left(X, G^{*}, \Delta\right)$ be a complete Menger PGM-space and let $\Delta$ be a continuous t-norm. Let $T: X \rightarrow X$ be a $(H, \psi, \Phi)$-contraction mapping satisfying (10). Then we have the following:

(i) $T$ is continuous on $X$;

(ii) Thas a unique fixed point in $X$, and, for any given $x_{0} \epsilon$ $X$, the iterative sequence $\left\{T^{n} x_{0}\right\}$ converges to this fixed point.

Proof. (i) Since $\Phi$ is right continuous, strictly increasing and $\Phi(0)=0$, we have $\lim _{\delta \rightarrow 0^{+}} \Phi(\delta)=0$ and $\Phi(t)>0$, for $t>$ 0 . Since $\lim _{s \rightarrow 1^{-}} \psi(s)=1$, we get $\lim _{\delta \rightarrow 0^{+}} \psi(1-\Phi(\delta))=1$. Hence, for any given $\varepsilon>0$ and $\lambda \in(0,1)$, there exists $\delta>0$, such that $0<\Phi(\delta)<\varepsilon$ and $\psi(1-\Phi(\delta))>1-\lambda$.

Now, for each $x \in X$, suppose that $\left\{x_{n}\right\}$ is convergent to $x$. Then, for $\delta>0$, there exists $M \in N$, such that $G_{x_{n}, x, x}^{*}(\delta)>$ $\psi(1-\delta)$, whenever $n \geq M$. Since $T$ is a $(H, \psi, \Phi)$-contraction mapping, we have $G_{T x_{n}, T x, T x}^{*}(\Phi(\delta))>\psi(1-\Phi(\delta))$, whenever $n \geq M$. Thus, $G_{T x_{n}, T x, T x}^{*}(\varepsilon) \geq G_{T x_{n}, T x, T x}^{*}(\Phi(\delta))>\psi(1-$ $\Phi(\delta))>1-\lambda$, which implies that $T$ is continuous at $x$. By the arbitrariness of $x$, we obtain that $T$ is continuous on $X$.

(ii) For any given $x_{0} \in X$, define the sequence $\left\{x_{n}\right\}$ by $x_{n}=T^{n} x_{0}$. Let $\varepsilon>0$. Then $G_{x_{0}, x_{m}, x_{m}}^{*}(1+\varepsilon) \geq 0>\psi(1-(1+\varepsilon))$, and it follows from (10) that $G_{T^{n} x_{0}, T^{n} x_{m}, T^{n} x_{m}}^{*}\left(\Phi^{n}(1+\varepsilon)\right)>\psi(1-$ $\left.\Phi^{n}(1+\varepsilon)\right)$ for $n, m \in Z^{+}$.

Since $\lim _{n \rightarrow \infty} \Phi^{n}(1+\varepsilon)=0$, we have $\lim _{n \rightarrow \infty} \psi\left(1-\Phi^{n}(1+\right.$ $\varepsilon))=1$. Hence, for any $\varepsilon>0$ and $\lambda>0$, there exists $M \in Z^{+}$ such that $\Phi^{n}(1+\varepsilon)<\varepsilon$ and $\psi\left(1-\Phi^{n}(1+\varepsilon)\right)>1-\lambda$, whenever 
$n \geq M$. Hence, for $n \geq M$, we have $G_{x_{n}, x_{n+m}, x_{n+m}}^{*}(\varepsilon)=$ $G_{T^{n} x_{0}, T^{n} x_{m}, T^{n} x_{m}}^{*}(\varepsilon) \geq \psi\left(1-\Phi^{n}(1+\varepsilon)\right)>1-\lambda$. Using Lemma 12, we know that $\left\{x_{n}\right\}$ is a Cauchy sequence. Since $\left(X, G^{*}\right)$ is complete, there exists $x^{*} \in X$ such that $x_{n} \rightarrow x^{*}$. Since $T$ is continuous, we have $T x^{*}=x^{*}$.

Now, Suppose that $y^{*}$ is another fixed point of $T$. Then, for any $\varepsilon>0$ and $\lambda>0, G_{x^{*}, y^{*}, y^{*}}^{*}(1+\varepsilon)>\psi(1-(1+\varepsilon))$, which implies that $G_{T^{n} x^{*}, T^{n} y^{*}, T^{n} y^{*}}^{*}\left(\Phi^{n}(1+\varepsilon)\right)>\psi\left(1-\Phi^{n}(1+\varepsilon)\right)$. For any given $\varepsilon>0$ and $\lambda>0$, there exists $M \in Z^{+}$such that $\Phi^{n}(1+\varepsilon)<\varepsilon$ and $\psi\left(1-\Phi^{n}(1+\varepsilon)\right)>1-\lambda$, whenever $n \geq M$. Hence, for $n \geq M$, we have $G_{x^{*}, y^{*}, y^{*}}^{*}(\varepsilon)=G_{T x^{*}, T y^{*}, T y^{*}}^{*}(\varepsilon) \geq$ $G_{T^{n} x^{*}, T^{n} y^{*}, T^{n} y^{*}}^{*}\left(\Phi^{n}(1+\varepsilon)\right)>1-\lambda$, which implies that $x^{*}=y^{*}$. This completes the proof.

Now, we present an example to illustrate Theorem 16.

Example 17. Let $X=[0,1]$. Define $G^{*}: X \times X \times X \rightarrow D^{+}$ by $G_{x, y, z}^{*}(t)=H(t-\max \{|x-y|,|y-z|,|x-z|\})$ for all $x, y, z \in X$ and $t \in R$. It is easy to verify that $\left(X, G^{*}, \Delta\right)$ is a complete Menger PGM-space. Let $\Phi(t)=(1 / 3) t$ for all $t \in R^{+}$, let $\psi(s)=s^{3}$ for all $s \in(-\infty, 1)$, and let $T: X \rightarrow X$ be defined by $T x=(1 / 4) x$.

Now, if $t>3$, then $G_{x, y, z}^{*}(t) \geq 0>(1-t)^{3}$ and $G_{T x, T y, T z}^{*}(t / 3) \geq 0>(1-t / 3)^{3}$ for all $x, y, z \in X$.

If $1<t \leq 3$, then $G_{x, y, z}^{*}(t)=1>(1-t)^{3}$. For any $x, y, z \in$ $X=[0,1]$, we have $\max \{|x-y|,|y-z|,|x-z|\} \leq 1$. Hence,

$$
\begin{aligned}
& G_{T x, T y, T z}^{*}\left(\frac{t}{3}\right) \\
& \quad=H\left(\frac{t}{3}-\max \left\{\frac{1}{4}|x-y|, \frac{1}{4}|y-z|, \frac{1}{4}|x-z|\right\}\right) \\
& =H\left(\frac{1}{3}\left[t-\frac{3}{4} \max \{|x-y|,|y-z|,|x-z|\}\right]\right) \\
& =H\left(t-\frac{3}{4} \max \{|x-y|,|y-z|,|x-z|\}\right) \\
& \quad \geq H\left(t-\frac{3}{4}\right) \geq H\left(1-\frac{3}{4}\right)=1>\left(1-\frac{t}{3}\right)^{3} .
\end{aligned}
$$

If $0<t \leq 1$ and $G_{x, y, z}^{*}(t)=H(t-\max \{|x-y|,|y-z|, \mid x-$ $z \mid\})>(1-t)^{3} \geq 0$, then $G_{x, y, z}^{*}(t)=1$ and

$$
\begin{aligned}
& G_{T x, T y, T z}^{*}\left(\frac{t}{3}\right) \\
& =H\left(t-\frac{3}{4} \max \{|x-y|,|y-z|,|x-z|\}\right) \\
& \geq H(t-\max \{|x-y|,|y-z|,|x-z|\}) \\
& =1>\left(1-\frac{t}{3}\right)^{3} .
\end{aligned}
$$

Thus, all the conditions of Theorem 16 are satisfied. Hence, $T$ has a unique fixed point in $X$. In fact, $x=0$ is the unique fixed point of $T$.
Remark 18. Theorem 16 generalizes and extends fixed point theorems for $H$-contraction mappings in [8] to the setting of generalized probabilistic metric spaces (take $\psi(s)=s$ ).

Definition 19. Let $T: X \rightarrow X$ be a given mapping and $\alpha: X^{3} \times(0, \infty) \rightarrow[0, \infty)$ be a function. We say that $T$ is generalized $\alpha$-admissible; if $x, y, z \in X$, for all $t>0$, $\alpha(x, y, z, t) \geq 1$, then $\alpha(T x, T y, T z, t) \geq 1$ for all $t>0$.

Definition 20. Let $\left(X, G^{*}\right)$ be a PGM-space and let $\varphi: R^{+} \rightarrow$ $R^{+}$be a strictly increasing function such that $\varphi^{-1}(\{0\})=\{0\}$ and $\sum_{n=1}^{\infty} \varphi^{n}(t)<+\infty$ for any $t>0$. A mapping $T: X \rightarrow$ $X$ is said to be a probabilistic $(\alpha, \varphi)$-contraction mapping on $\left(X, G^{*}\right)$ if there exists a function $\alpha: X^{3} \times(0, \infty) \rightarrow[0, \infty)$, for each $x, y, z \in X, t>0$,

$$
G_{T x, T y, T z}^{*}(\varphi(t)) \geq \alpha(x, y, z, t) U_{x, y, z}(t)
$$

where $U_{x, y, z}(t)=\min \left\{G_{x, y, z}^{*}(t), G_{x, T x, T x}^{*}(t), G_{y, T y, T y}^{*}(t)\right.$, $\left.G_{z, T z, T z}^{*}(t), G_{x, x, T x}^{*}(t), G_{y, y, T y}^{*}(t), G_{z, z, T z}^{*}(t)\right\}$.

Theorem 21. Let $\left(X, G^{*}, \Delta\right)$ be a complete Menger PGM-space such that $\Delta$ is a t-norm of $H$-type and $\Delta \geq \Delta_{1}$. Let $T: X \rightarrow$ $X$ be a probabilistic $(\alpha, \varphi)$-contraction mapping satisfying (13). Suppose that the following hold:

\section{(i) $T$ is generalized $\alpha$-admissible;}

(ii) there exists $x_{0} \in X$ such that $\alpha\left(x_{0}, T x_{0}, T x_{0}, t\right) \geq 1$ and $\alpha\left(x_{0}, x_{0}, T x_{0}, t\right) \geq 1$ for all $t>0$;

(iii) if $\left\{x_{n}\right\}$ is a sequence in $X$ such that $\alpha\left(x_{n}, x_{n+1}, x_{n+1}, t\right) \geq$ $1, \alpha\left(x_{n}, x_{n}, x_{n+1}, t\right) \geq 1$ for all $n \in N, t>0$, and $x_{n} \rightarrow$ $x$, then $\alpha\left(x_{n}, x, x, t\right) \geq 1$ and $\alpha\left(x_{n}, x_{n}, x, t\right) \geq 1$ for all $n \in N$ and $t>0$.

Then $T$ has a fixed point in X. Moreover, let $A$ be the set of fixed points of $T$; if, for each $x, y \in A$, we have $\alpha(x, y, y, t) \geq 1$ for all $t>0$, then $T$ has a unique fixed point in $X$.

Proof. Let $x_{0} \in X$ and define the sequence $\left\{x_{n}\right\}$ by $x_{n}=T^{n} x_{0}$. Suppose that $x_{n} \neq x_{n+1}$, for any $n \in N$ (If not, there exists $n_{0} \in N$, such that $x_{n_{0}+1}=T x_{n_{0}}=x_{n_{0}}$ and then the conclusion holds).

Since $T$ is $\alpha$-admissible, $\alpha\left(x_{0}, T x_{0}, T x_{0}, t\right) \geq 1$, and $\alpha\left(x_{0}, x_{0}, T x_{0}, t\right) \geq 1$ for all $t>0$, we have $\alpha\left(x_{1}, x_{2}, x_{2}, t\right) \geq 1$ and $\alpha\left(x_{1}, x_{1}, x_{2}, t\right) \geq 1$ for all $t>0$. By induction, we obtain that

$$
\begin{array}{r}
\alpha\left(x_{n}, x_{n+1}, x_{n+1}, t\right) \geq 1, \quad \alpha\left(x_{n}, x_{n}, x_{n+1}, t\right) \geq 1 \\
\forall n \in N, \quad t>0 .
\end{array}
$$


For any $n \in N$, from (13) and (14), we have

$$
\begin{aligned}
& G_{x_{n}, x_{n+1}, x_{n+1}}^{*}(\varphi(t)) \\
& \geq \alpha\left(x_{n-1}, x_{n}, x_{n}, t\right) \min \left\{G_{x_{n-1}, x_{n}, x_{n}}^{*}(t), G_{x_{n-1}, x_{n}, x_{n}}^{*}(t),\right. \\
& G_{x_{n}, x_{n+1}, x_{n+1}}^{*}(t), G_{x_{n}, x_{n+1}, x_{n+1}}^{*}(t), \\
& G_{x_{n-1}, x_{n-1}, x_{n}}^{*}(t), G_{x_{n}, x_{n}, x_{n+1}}^{*}(t) \text {, } \\
& \left.G_{x_{n}, x_{n}, x_{n+1}}^{*}(t)\right\} \\
& \geq \min \left\{G_{x_{n-1}, x_{n}, x_{n}}^{*}(t), G_{x_{n}, x_{n+1}, x_{n+1}}^{*}(t), G_{x_{n-1}, x_{n-1}, x_{n}}^{*}(t)\right. \text {, } \\
& \left.G_{x_{n}, x_{n}, x_{n+1}}^{*}(t)\right\}, \\
& G_{x_{n}, x_{n}, x_{n+1}}^{*}(\varphi(t)) \\
& \geq \alpha\left(x_{n-1}, x_{n-1}, x_{n}, t\right) \min \left\{G_{x_{n-1}, x_{n-1}, x_{n}}^{*}(t), G_{x_{n-1}, x_{n}, x_{n}}^{*}(t),\right. \\
& G_{x_{n-1}, x_{n}, x_{n}}^{*}(t), G_{x_{n}, x_{n+1}, x_{n+1}}^{*}(t), \\
& G_{x_{n-1}, x_{n-1}, x_{n}}^{*}(t), G_{x_{n-1}, x_{n-1}, x_{n}}^{*}(t) \text {, } \\
& \left.G_{x_{n}, x_{n}, x_{n+1}}^{*}(t)\right\} \\
& \geq \min \left\{G_{x_{n-1}, x_{n}, x_{n}}^{*}(t), G_{x_{n}, x_{n+1}, x_{n+1}}^{*}(t), G_{x_{n-1}, x_{n-1}, x_{n}}^{*}(t),\right. \\
& \left.G_{x_{n}, x_{n}, x_{n+1}}^{*}(t)\right\} \text {. }
\end{aligned}
$$

Suppose that $Q_{n}(t)=\min \left\{G_{x_{n}, x_{n+1}, x_{n+1}}^{*}(t), G_{x_{n}, x_{n}, x_{n+1}}^{*}(t)\right\}$. Then, from the above inequalities, we get $Q_{n}(\varphi(t)) \geq$ $\min \left\{Q_{n}(t), Q_{n-1}(t)\right\}$. If $\min \left\{Q_{n}(t), Q_{n-1}(t)\right\}=Q_{n}(t)$, then

$$
Q_{n}\left(\varphi^{n}(t)\right) \geq Q_{n}\left(\varphi^{n-1}(t)\right) \geq \cdots \geq Q_{n}(t) .
$$

By Lemma 10, we have $Q_{n}(t)=1$ for any $t>0$. Then, $x_{n}=$ $x_{n+1}$, which is in contradiction to $x_{n} \neq x_{n+1}$.

Hence, $\min \left\{Q_{n}(t), Q_{n-1}(t)\right\}=Q_{n-1}(t)$. Then we have

$$
Q_{n}\left(\varphi^{n}(t)\right) \geq Q_{n-1}\left(\varphi^{n-1}(t)\right) \geq \cdots \geq Q_{0}(t)
$$

Next, we show that $\left\{x_{n}\right\}$ is a Cauchy sequence. For every $\lambda \in(0,1]$, suppose that $D_{\lambda}=\inf \left\{t>0: Q_{0}(t)>1-\lambda\right\}$. Then $Q_{0}\left(D_{\lambda}+1\right)>1-\lambda$. From (17), for every $\lambda \in(0,1]$, we get $Q_{n}\left(\varphi^{n}\left(D_{\lambda}+1\right)\right)>1-\lambda$. By Lemma 13 , for any $n \in Z^{+}$, we have

$$
\begin{gathered}
G_{\lambda}^{*}\left(x_{n}, x_{n+1}, x_{n+1}\right)<\varphi^{n}\left(D_{\lambda}+1\right), \\
G_{\lambda}^{*}\left(x_{n}, x_{n}, x_{n+1}\right)<\varphi^{n}\left(D_{\lambda}+1\right) .
\end{gathered}
$$

By Lemma 14, for every $\lambda \in(0,1]$, there exists $\mu \in(0, \lambda]$ such that

$$
G_{\lambda}^{*}\left(x_{n}, x_{n+m}, x_{n+m}\right) \leq \sum_{i=n}^{m+n-1} G_{\mu}^{*}\left(x_{i}, x_{i+1}, x_{i+1}\right),
$$

$\forall n, m \in Z^{+}$
Suppose that $\varepsilon>0$ and $\lambda \in(0,1]$ are given. Since $\sum_{n=1}^{\infty} \varphi^{n}\left(D_{\mu}+1\right)<+\infty$, there exists $N \in Z^{+}$, such that $\sum_{i=n}^{m+n-1} \varphi^{i}\left(D_{\mu}+1\right)<\varepsilon$ for all $n, m \geq N$. Thus, by (18) and (19), we have $G_{\lambda}^{*}\left(x_{n}, x_{n+m}, x_{n+m}\right)<\varepsilon$. Using Lemma 13, we have $G_{x_{n}, x_{n+m}, x_{n+m}}^{*}(\varepsilon)>1-\lambda$ for all $n, m \geq N$. From Lemma 12, we know that $\left\{x_{n}\right\}$ is a Cauchy sequence. Since $X$ is complete, there exists $x^{*} \in X$, such that $x_{n} \rightarrow x^{*}$.

Since $x_{n} \rightarrow x^{*}$, by (14) and (iii), we have $\alpha\left(x_{n}, x^{*}, x^{*}, t\right) \geq 1$ and $\alpha\left(x_{n}, x_{n}, x^{*}, t\right) \geq 1$ for all $n \in N$ and $t>0$. From (13), for any $n \in N$, we have

$$
\begin{aligned}
& G_{x_{n}, T x^{*}, T x^{*}}^{*}(\varphi(t)) \\
& \geq \alpha\left(x_{n-1}, x^{*}, x^{*}, t\right) \min \left\{G_{x_{n-1}, x^{*}, x^{*}}^{*}(t), G_{x_{n-1}, x_{n}, x_{n}}^{*}(t),\right. \\
& G_{x^{*}, T x^{*}, T x^{*}}^{*}(t), G_{x^{*}, T x^{*}, T x^{*}}^{*}(t), \\
& G_{x_{n-1}, x_{n-1}, x_{n}}^{*}(t), G_{x^{*}, x^{*}, T x^{*}}^{*}(t), \\
& \left.G_{x^{*}, x^{*}, T x^{*}}^{*}(t)\right\} \\
& \geq \min \left\{G_{x_{n-1}, x^{*}, x^{*}}^{*}(t), G_{x_{n-1}, x_{n}, x_{n}}^{*}(t), G_{x_{n-1}, x_{n-1}, x_{n}}^{*}(t),\right. \\
& \left.G_{x^{*}, T x^{*}, T x^{*}}^{*}(t), G_{x^{*}, x^{*}, T x^{*}}^{*}(t)\right\}, \\
& G_{x_{n}, x_{n}, T x^{*}}^{*}(\varphi(t)) \\
& \geq \alpha\left(x_{n-1}, x_{n-1}, x^{*}, t\right) \min \left\{G_{x_{n-1}, x_{n-1}, x^{*}}^{*}(t), G_{x_{n-1}, x_{n}, x_{n}}^{*}(t),\right. \\
& G_{x_{n-1}, x_{n}, x_{n}}^{*}(t), G_{x^{*}, T x^{*}, T x^{*}}^{*}(t), \\
& G_{x_{n-1}, x_{n-1}, x_{n}}^{*}(t), G_{x_{n-1}, x_{n-1}, x_{n}}^{*}(t) \text {, } \\
& \left.G_{x^{*}, x^{*}, T x^{*}}^{*}(t)\right\} \\
& \geq \min \left\{G_{x_{n-1}, x^{*}, x^{*}}^{*}(t), G_{x_{n-1}, x_{n}, x_{n}}^{*}(t), G_{x_{n-1}, x_{n-1}, x_{n}}^{*}(t)\right. \text {, } \\
& \left.G_{x^{*}, T x^{*}, T x^{*}}^{*}(t), G_{x^{*}, x^{*}, T x^{*}}^{*}(t)\right\} .
\end{aligned}
$$

Letting $n \rightarrow \infty$, since $x_{n} \rightarrow x^{*}$ as $n \rightarrow \infty$, by Lemma 11, (20), we have

$$
\begin{aligned}
& \min \left\{G_{x^{*}, T x^{*}, T x^{*}}^{*}(\varphi(t)), G_{x^{*}, x^{*}, T x^{*}}^{*}(\varphi(t))\right\} \\
& \geq \min \left\{1,1,1, G_{x^{*}, T x^{*}, T x^{*}}^{*}(t), G_{x^{*}, x^{*}, T x^{*}}^{*}(t)\right\} .
\end{aligned}
$$

Hence,

$$
\begin{aligned}
& \min \left\{G_{x^{*}, T x^{*}, T x^{*}}\left(\varphi^{n}(t)\right), G_{x^{*}, x^{*}, T x^{*}}\left(\varphi^{n}(t)\right)\right\} \\
& \geq \min \left\{G_{x^{*}, T x^{*}, T x^{*}}\left(\varphi^{n-1}(t)\right), G_{x^{*}, x^{*}, T x^{*}}\left(\varphi^{n-1}(t)\right)\right\} \\
& \geq \cdots \geq \min \left\{G_{x^{*}, T x^{*}, T x^{*}}(t), G_{x^{*}, x^{*}, T x^{*}}(t)\right\} .
\end{aligned}
$$

By Lemma 10, we have $G_{x^{*}, T x^{*}, T x^{*}}^{*}(t)=1$ and $G_{x^{*}, x^{*}, T x^{*}}^{*}(t)=1$ for all $t>0$. Thus $T x^{*}=x^{*}$.

Moreover, let $A$ be the set of fixed points of $T$; if for each $x, y \in A$, we have $\alpha(x, y, y, t) \geq 1$ for all $t>0$. Now, suppose 
that $y^{*}$ is another fixed point of $T$. Then $\alpha\left(x^{*}, y^{*}, y^{*}, t\right) \geq 1$ for all $t>0$. Hence, by (13), we have

$$
\begin{aligned}
& G_{x^{*}, y^{*}, y^{*}}^{*}\left(\varphi^{n}(t)\right) \\
& =G_{T x^{*}, T y^{*}, T y^{*}}^{*}\left(\varphi^{n}(t)\right) \\
& \geq \alpha\left(x^{*}, y^{*}, y^{*}, t\right) \\
& \quad \times \min \left\{G_{x^{*}, y^{*}, y^{*}}^{*}\left(\varphi^{n-1}(t)\right), G_{x^{*}, T x^{*}, T x^{*}}^{*}\left(\varphi^{n-1}(t)\right),\right. \\
& G_{y^{*}, T y^{*}, T y^{*}}^{*}\left(\varphi^{n-1}(t)\right), G_{y^{*}, T y^{*}, T y^{*}}^{*}\left(\varphi^{n-1}(t)\right), \\
& G_{x^{*}, x^{*}, T x^{*}}^{*}\left(\varphi^{n-1}(t)\right), G_{y^{*}, y^{*}, T y^{*}}^{*}\left(\varphi^{n-1}(t)\right), \\
& \left.G_{y^{*}, y^{*}, T y^{*}}^{*}\left(\varphi^{n-1}(t)\right)\right\} \\
& \geq \min _{\geq}\left\{G_{x^{*}, y^{*}, y^{*}}^{*}\left(\varphi^{n-1}(t)\right), 1,1,1,1,1,1\right\} \\
& G_{x^{*}, y^{*}, y^{*}}^{*}\left(\varphi^{n-1}(t)\right) \geq \cdots \geq G_{x^{*}, y^{*}, y^{*}}^{*}(t) .
\end{aligned}
$$

By Lemma 10, we get $x^{*}=y^{*}$. This completes the proof.

Now, we present an example to illustrate Theorem 21.

Example 22. Let $X=[0,+\infty]$ and $\Delta=\Delta_{M}$. Then $\Delta$ is a $t$ norm of $H$-type. Define $G^{*}: X \times X \times X \rightarrow D^{+}$by

$$
G_{x, y, z}^{*}(t)= \begin{cases}0, & t \leq 0 \\ e^{-\max \{|x-y|,|y-z|,|x-z|\} / t}, & t>0\end{cases}
$$

for all $x, y, z \in X$. We claim that $\left(X, G^{*}, \Delta_{M}\right)$ is a Menger PGM-space. In fact, (PGM - 1)-(PGM - 3) are easy to check. Next, we prove that $(\mathrm{PGM}-4)$ holds.

Suppose that $t, s>0, x, y, z, a \in X$, and $\Delta_{M}\left(G_{x, a, a}^{*}(t)\right.$, $\left.G_{a, y, z}^{*}(s)\right)=\min \left\{e^{-|x-a| / t}, e^{-\max \{|a-y|,|y-z|,|a-z|\} / s}\right\}=e^{-|x-a| / t}$. Then we have $(s / t)|x-a| \geq \max \{|a-y|,|y-z|,|a-z|\}$ and so $(t+s) / t|x-a|=|x-a|+(s / t)|x-a| \geq|x-a|+$ $\max \{|a-y|,|y-z|,|a-z|\} \geq \max \{|x-y|,|y-z|,|x-z|\}$, which implies that $G_{x, y, z}^{*}(t+s)=e^{-\max \{|x-y|,|y-z|,|x-z|\} /(t+s)} \geq$ $e^{-|x-a| / t}=\Delta_{M}\left(G_{x, a, a}^{*}(t), G_{a, y, z}^{*}(s)\right)$.

Similarly, if $\Delta_{M}\left(G_{x, a, a}^{*}(t), G_{a, y, z}^{*}(s)\right)=G_{a, y, z}^{*}(s)$, then we also have $G_{x, y, z}^{*}(t+s)=\Delta_{M}\left(G_{x, a, a}^{*}(t), G_{a, y, z}^{*}(s)\right)$. Hence, (PGM - 4) holds. It is easy to prove that $\left(X, G^{*}, \Delta_{M}\right)$ is complete. Let $\varphi(t)=t / 2$ for $t \in R^{+}$,

$$
\begin{gathered}
T x= \begin{cases}\frac{x}{3}+\frac{1}{3}, & x \in[0,2], \\
x^{2}, & x \in(2, \infty),\end{cases} \\
\alpha(x, y, z, t)= \begin{cases}1, & x, y, z \in[0,2], \\
0, & \text { otherwise. }\end{cases}
\end{gathered}
$$

It is not difficult to prove that $\alpha$ and $T$ satisfy (i), (ii), and (iii) of Theorem 21. Now, suppose that at least one of $x, y, z$ is in $(2, \infty)$. Then $\alpha(x, y, z, t)=0$ and so inequality (13) holds.
Hence, for $x, y, z \in[0,2]$, we have $\alpha(x, y, z, t)=1$ for all $t>0$. Then, for $t>0$, we have

$$
\begin{aligned}
G_{T x, T y, T z}^{*}\left(\frac{t}{2}\right) \\
\quad=e^{-(1 / 3) \max \{|x-y|,|y-z|,|x-z|\} /(1 / 2) t} \\
=e^{-(2 / 3) \max \{|x-y|,|y-z|,|x-z|\} / t} \\
\geq e^{-\max \{|x-y|,|y-z|,|x-z|\} / t} \\
=\alpha(x, y, z, t) G_{x, y, z}^{*}(t) .
\end{aligned}
$$

Thus, all the conditions of Theorem 21 are satisfied. Hence, $T$ has a unique fixed point in $X$. In fact, $x=1 / 2$ is the unique fixed point of $T$ in $X$.

Remark 23. Theorem 21 generalizes and extends Theorem 4.2 in [16] and Theorems 3.5 and 3.6 in [17] (take $\alpha(x, y, z, t) \equiv 1$ and $t>0)$.

\section{An Application}

In this section, we will apply one of our main results to investigate the existence of solutions for a class of integral equations.

Consider the following class of integral equations:

$$
x(t)=\int_{0}^{T_{0}} K(t, s) f(t, s, x(s)) d s+x_{0}(t),
$$

where $t \in I=\left[0, T_{0}\right], T_{0}>0$, and $x_{0}:\left[0, T_{0}\right] \rightarrow R, K$ : $\left[0, T_{0}\right]^{2} \rightarrow R^{+}$, and $f:\left[0, T_{0}\right]^{2} \times R \rightarrow R^{+}$are all continuous functions.

Let $X=C\left(\left[0, T_{0}\right], R\right)$ be the set of all real continuous functions defined on $\left[0, T_{0}\right]$. We define $G^{*}: X \times X \times X \rightarrow D^{+}$ by

$$
\begin{aligned}
& G_{x, y, z}^{*}(t) \\
& =H\left(t-\max \left\{\sup _{t \in\left[0, T_{0}\right]}|x(t)-y(t)|, \sup _{t \in\left[0, T_{0}\right]}|y(t)-z(t)|,\right.\right. \\
& \left.\left.\sup _{t \in\left[0, T_{0}\right]}|x(t)-z(t)|\right\}\right),
\end{aligned}
$$

for all $x, y, z \in X$ and $t \in R$. It is easy to verify that $\left(X, G^{*}, \Delta_{M}\right)$ is a complete Menger PGM-space.

Now, we define $T: X \rightarrow X$ by $T x(t)=$ $\int_{0}^{T_{0}} K(t, s) f(t, s, x(s)) d s+x_{0}(t)$, for $t \in\left[0, T_{0}\right]$ and we write $x \leq y$ if and only if $x(t) \leq y(t)$ for all $t \in\left[0, T_{0}\right]$. Then, $x$ is a solution of (27) if and only if it is a fixed point of $T$. 
Theorem 24. Suppose that the following hypotheses hold:

(i) $f(t, s, \cdot)$ is nondecreasing and $\varphi: R^{+} \rightarrow R^{+}$is a strictly increasing function such that $\varphi^{-1}(\{0\})=\{0\}$ and $\sum_{n=1}^{\infty} \varphi^{n}(t)<+\infty$ for any $t>0$;

(ii) one of the following conditions is satisfied:

(a) for all $x, y \in X, x \leq y$, and $\varepsilon>0$, we have

$$
\begin{array}{r}
\sup _{t \in\left[0, T_{0}\right]} \int_{0}^{T_{0}} K(t, s)[f(t, s, y(s))-f(t, s, x(s))] d s<\varphi(\varepsilon), \\
\text { whenever } \sup _{t \in\left[0, T_{0}\right]}[y(t)-x(t)]<\varepsilon ;
\end{array}
$$

(b) there exists a continuous function $L:\left[0, T_{0}\right]^{2} \rightarrow$ $R^{+}$such that

$$
\begin{array}{r}
\frac{\varepsilon}{T_{0}}+K(t, s)[f(t, s, v)-f(t, s, u)] \leq \frac{\varphi(\varepsilon)}{T_{0}}+L(t, s)(v-u) \\
\forall u, v \in R, \quad u \leq v, \quad t, s \in\left[0, T_{0}\right]
\end{array}
$$

(iii) $\sup _{t \in\left[0, T_{0}\right]} \int_{0}^{T_{0}} L(t, s) d s \leq 1$.

Then the integral equation (27) has a solution in $X$.

Proof. Since $\Delta=\Delta_{M}, \Delta$ is a $t$-norm of $H$-type and $\Delta \geq \Delta_{1}$. Let

$$
\alpha(x, y, z, t)= \begin{cases}1, & x, y, z \in X, x \leq y \leq z \\ 0, & \text { otherwise }\end{cases}
$$

If $x, y, z \in X$, for all $t>0, \alpha(x, y, z, t) \geq 1$, then $x \leq y \leq z$. Since $f(t, s, \cdot)$ is nondecreasing, we have $T x \leq T y \leq T z$. Hence, $\alpha(T x, T y, T z, t) \geq 1$. Thus, $T$ is generalized $\alpha$-admissible. Let $y_{0}=x_{0} \in X$. Then $y_{0} \leq T y_{0}$. Hence, $\alpha\left(y_{0}, y_{0}, T y_{0}, t\right) \geq 1$ and $\alpha\left(y_{0}, T y_{0}, T y_{0}, t\right) \geq 1$ for all $t>0$. Suppose that $\left\{y_{n}\right\}$ is a sequence in $X$ such that $\alpha\left(y_{n}, y_{n+1}, y_{n+1}, t\right) \geq 1$ and $\alpha\left(y_{n}, y_{n}, y_{n+1}, t\right) \geq 1$ for all $n \in N$, $t>0$, and $y_{n} \rightarrow y$. Then $y_{0} \leq y_{1} \leq \cdots \leq y_{n} \leq y_{n+1} \leq \cdots \leq$ $y$. Thus, $\alpha\left(y_{n}, y_{n}, y, t\right) \geq 1$ and $\alpha\left(y_{n}, y, y, t\right) \geq 1$ for all $n \in N$ and $t>0$.

We now prove that $G_{T x, T y, T z}^{*}(\varphi(\varepsilon)) \geq \alpha(x, y, z, \varepsilon) G_{x, y, z}^{*}(\varepsilon)$, for $\varepsilon>0$. Suppose that $x \nless y$ or $y \not z$. Then $\alpha(x, y, z, \varepsilon)=0$. Hence, the above inequality holds.

If $x \leq y \leq z$, then $\alpha(x, y, z, \varepsilon)=1$ for all $\varepsilon>0$. Here we distinguish two cases.

Case (a). If $\max \left\{\sup _{t \in\left[0, T_{0}\right]}[y(t)-x(t)], \sup _{t \in\left[0, T_{0}\right]}[z(t)-\right.$ $\left.y(t)], \sup _{t \in\left[0, T_{0}\right]}[z(t)-x(t)]\right\} \geq \varepsilon$, then $G_{T x, T y, T z}^{*}(\varphi(\varepsilon)) \geq 0=$ $\alpha(x, y, z, \varepsilon) G_{x, y, z}^{*}(\varepsilon)$.

If $\max \left\{\sup _{t \in\left[0, T_{0}\right]}[y(t)-x(t)], \sup _{t \in\left[0, T_{0}\right]}[z(t)-y(t)]\right.$, $\left.\sup _{t \in\left[0, T_{0}\right]}[z(t)-x(t)]\right\}<\varepsilon$, then we have $\sup _{t \in\left[0, T_{0}\right]}[y(t)-$ $x(t)]<\varepsilon, \sup _{t \in\left[0, T_{0}\right]}[z(t)-y(t)]<\varepsilon$, and $\sup _{t \in\left[0, T_{0}\right]}[z(t)-$ $x(t)]<\varepsilon$. By (ii), we get $\sup _{t \in\left[0, T_{0}\right]}[T y(t)-T x(t)]<\varphi(\varepsilon)$, $\sup _{t \in\left[0, T_{0}\right]}[T z(t)-T y(t)]<\varphi(\varepsilon)$, and $\sup _{t \in\left[0, T_{0}\right]}[T z(t)-$ $T x(t)]<\varphi(\varepsilon)$. Hence, $G_{T x, T y, T z}^{*}(\varphi(\varepsilon))=1=\alpha(x, y, z, \varepsilon)$ $G_{x, y, z}^{*}(\varepsilon)$.

Case (b). For any $x, y \in X, x \leq y$, and $\varepsilon>0$, we have

$$
\begin{aligned}
& \varepsilon+\sup _{t \in\left[0, T_{0}\right]} T y(t)-T x(t) \\
& =\int_{0}^{T_{0}} \frac{\varepsilon}{T_{0}} d s \\
& \quad+\sup _{t \in\left[0, T_{0}\right]} \int_{0}^{T_{0}} K(t, s)(f(t, s, y(s))-f(t, s, x(s))) d s \\
& =\sup _{t \in\left[0, T_{0}\right]} \int_{0}^{T_{0}} \frac{\varepsilon}{T_{0}}+K(t, s)[f(t, s, y(s))-f(t, s, x(s))] d s \\
& \leq \sup _{t \in\left[0, T_{0}\right]} \int_{0}^{T_{0}} \frac{\varphi(\varepsilon)}{T_{0}}+L(t, s)[y(s)-x(s)] d s \\
& \leq \varphi(\varepsilon)+\sup _{t \in\left[0, T_{0}\right]}[y(t)-x(t)] \sup _{t \in\left[0, T_{0}\right]} \int_{0}^{T_{0}} L(t, s) d s \\
& \leq \varphi(\varepsilon)+\sup _{t \in\left[0, T_{0}\right]}[y(t)-x(t)] .
\end{aligned}
$$

Hence, $\varphi(\varepsilon)-\sup _{t \in\left[0, T_{0}\right]}[T y(t)-T x(t)] \geq \varepsilon-\sup _{t \in\left[0, T_{0}\right]}[y(t)-$ $x(t)$, for all $x, y \in X, x \leq y$, and $\varepsilon>0$. Then,

$$
\begin{gathered}
\varphi(\varepsilon)-\max \left\{\sup _{t \in\left[0, T_{0}\right]}[T y(t)-T x(t)], \sup _{t \in\left[0, T_{0}\right]}[T z(t)-T y(t)],\right. \\
\left.\sup _{t \in\left[0, T_{0}\right]}[T z(t)-T x(t)]\right\} \\
\geq \varepsilon-\max \left\{\sup _{t \in\left[0, T_{0}\right]}[y(t)-x(t)], \sup _{t \in\left[0, T_{0}\right]}[z(t)-y(t)],\right. \\
\left.\sup _{t \in\left[0, T_{0}\right]}[z(t)-x(t)]\right\},
\end{gathered}
$$

where $x \leq y \leq z$. Thus, $G_{T x, T y, T z}^{*}(\varphi(\varepsilon)) \geq \alpha(x, y, z, \varepsilon)$ $G_{x, y, z}^{*}(\varepsilon)$, for $\varepsilon>0$.

In all case, we obtain $G_{T x, T y, T z}^{*}(\varphi(t)) \geq \alpha(x, y, z, t)$ $G_{x, y, z}^{*}(t) \geq \alpha(x, y, z, t) U_{x, y, z}(t)$, for all $t>0$, where $U_{x, y, z}(t)$ is the same as the one in Definition 20. Thus, all the required conditions of Theorem 21 are satisfied. Therefore, $T$ has a fixed point $x^{*} \in X$; that is, $x^{*}$ is a solution of the integral equation (27). 


\section{Conflict of Interests}

The authors declare that there is no conflict of interests regarding the publication of this paper.

\section{Authors' Contribution}

All authors contributed equally and significantly in writing this paper. All authors read and approved the final paper.

\section{Acknowledgments}

The authors would like to thank the editor and the referees for their valuable comments and suggestions. The research was supported by the National Natural Science Foundation of China (11361042, 11326099, 11461045, and 11071108) and the Provincial Natural Science Foundation of Jiangxi, China (20132BAB201001, 20142BAB211016, and 2010GZS0147).

\section{References}

[1] K. Menger, "Statistical metrics," Proceedings of the National Academy of Sciences of the United States of America, vol. 28, pp. 535-537, 1942.

[2] B. Schweizer and A. Sklar, Probabilistic Metric Spaces, NorthHolland, Amsterdam, The Netherlands, 1983.

[3] O. Hadžić and E. Pap, Fixed Point Theory in Probabilistic Metric Spaces, Kluwer Academic, Dordrecht, The Netherlands, 2001.

[4] S. S. Chang, Y. J. Cho, and S. M. Kang, Probabilistic Metric Spaces and Nonlinear Operator Theory, Sichuan University Press, Chengdu, China, 1994.

[5] J. X. Fang and Y. Gao, "Common fixed point theorems under strict contractive conditions in Menger spaces," Nonlinear Analysis, vol. 70, no. 1, pp. 184-193, 2009.

[6] J. Jachymski, "On probabilistic $\varphi$-contractions on Menger spaces," Nonlinear Analysis: Theory, Methods \& Applications, vol. 73, no. 7, pp. 2199-2203, 2010.

[7] J.-Z. Xiao, X.-H. Zhu, and Y.-F. Cao, "Common coupled fixed point results for probabilistic $\varphi$-contractions in Menger spaces," Nonlinear Analysis: Theory, Methods \& Applications, vol. 74, no. 13, pp. 4589-4600, 2011.

[8] T. L. Hicks, "On the theory of fixed points in probabilistic metric spaces," Novi Sad Journal of Mathematics: Review of Research Faculty of Science, Mathematics Series, vol. 13, pp. 69-80, 1983.

[9] Z. Mustafa and B. Sims, "A new approach to generalized metric spaces," Journal of Nonlinear and Convex Analysis, vol. 7, no. 2, pp. 289-297, 2006.

[10] Z. Mustafa, H. Obiedat, and F. Awawdeh, "Some fixed point theorem for mapping on complete G-metric spaces," Fixed Point Theory and Applications, vol. 2008, Article ID 189870, 12 pages, 2008.

[11] Z. Mustafa and B. Sims, "Fixed point theorems for contractive mappings in complete G-metric spaces," Fixed Point Theory and Applications, vol. 2009, Article ID 917175, 2009.

[12] Z. Mustafa, F. Awawdeh, and W. Shatanawi, "Fixed point theorem for expansive mappings in $G$-metric spaces," International Journal of Contemporary Mathematical Sciences, vol. 5, no. 4952, pp. 2463-2472, 2010.

[13] R. Saadati, S. M. Vaezpour, P. Vetro, and B. . Rhoades, "Fixed point theorems in generalized partially ordered G-metric spaces," Mathematical and Computer Modelling, vol. 52, no. 56, pp. 797-801, 2010.

[14] W. Shatanawi, "Fixed point theory for contractive mappings satisfying $\phi$-maps in G-metric spaces," Fixed Point Theory and Applications, vol. 2010, Article ID 181650, 9 pages, 2010.

[15] W. Shatanawi, "Some fixed point theorems in ordered G-metric spaces and applications," Abstract and Applied Analysis, vol. 2011, Article ID 126205, 11 pages, 2011.

[16] M. Abbas, T. Nazir, and R. Saadati, "Common fixed point results for three maps in generalized metric space," Advances in Difference Equations, vol. 2011, article 49, 2011.

[17] C. Zhou, S. Wang, L. Ćirić, and S. M. Alsulami, "Generalized probabilistic metric spaces and fixed point theorems," Fixed Point Theory and Applications, vol. 2014, article 91, 2014. 


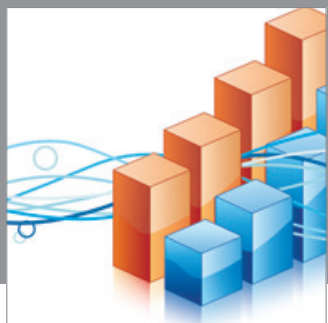

Advances in

Operations Research

mansans

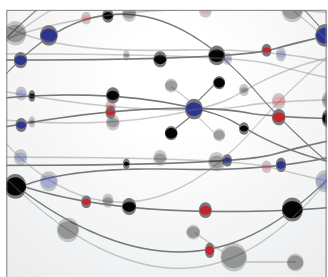

The Scientific World Journal
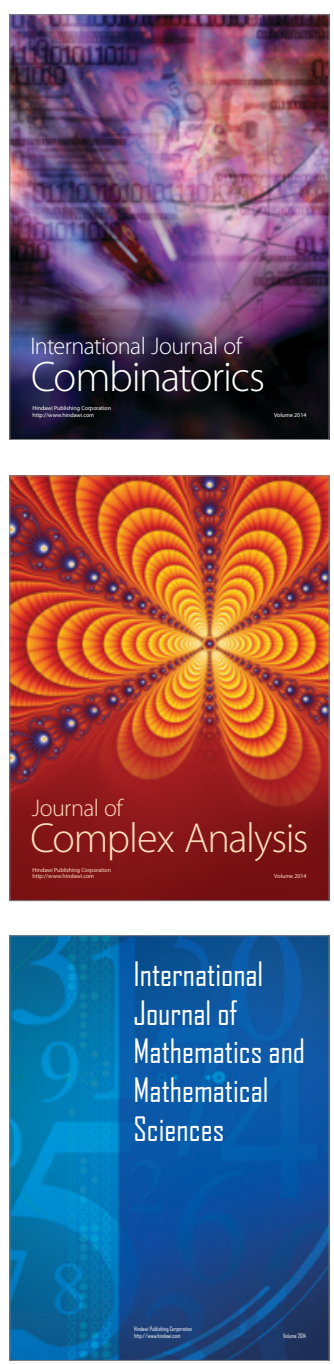
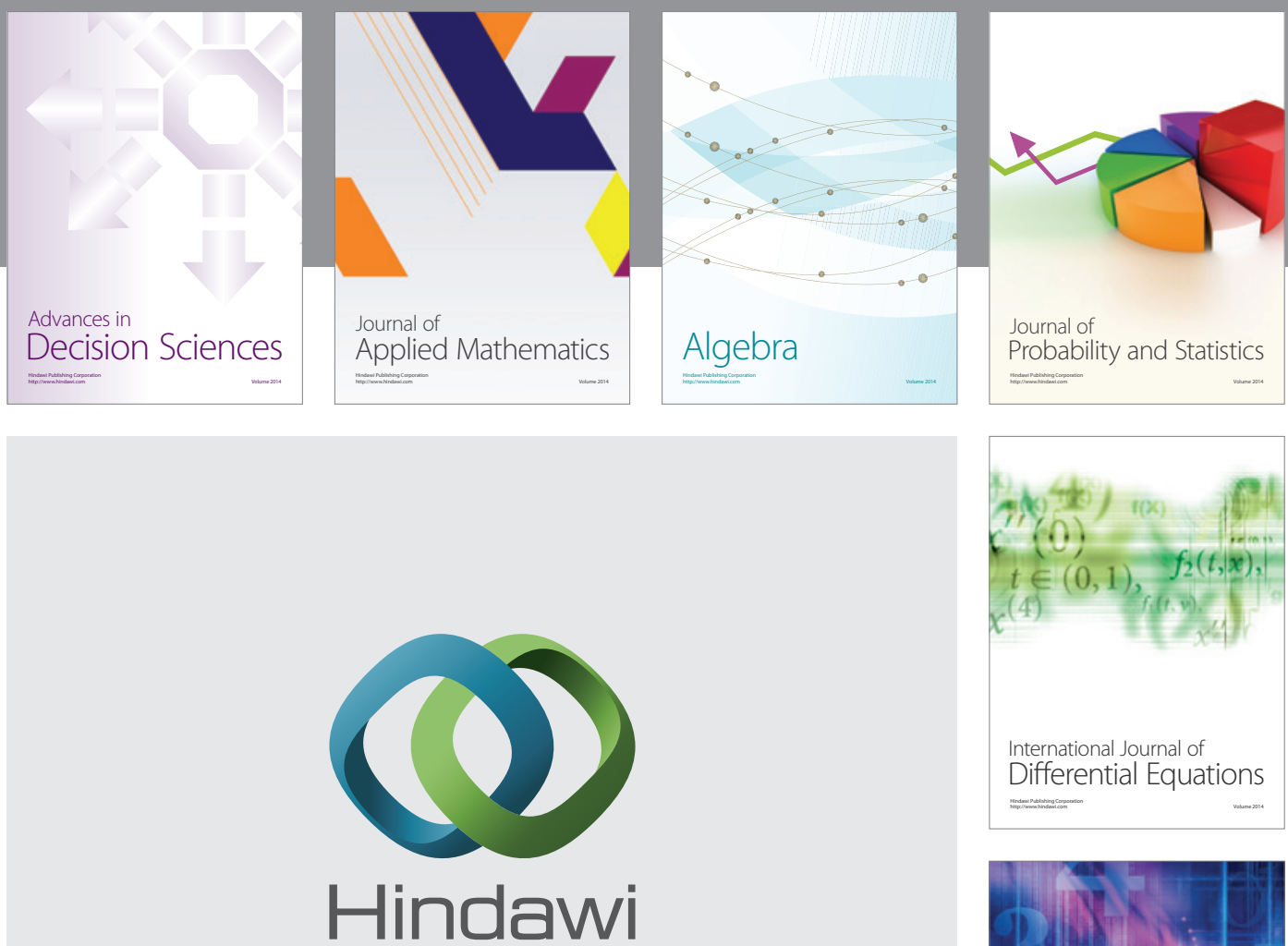

Submit your manuscripts at http://www.hindawi.com
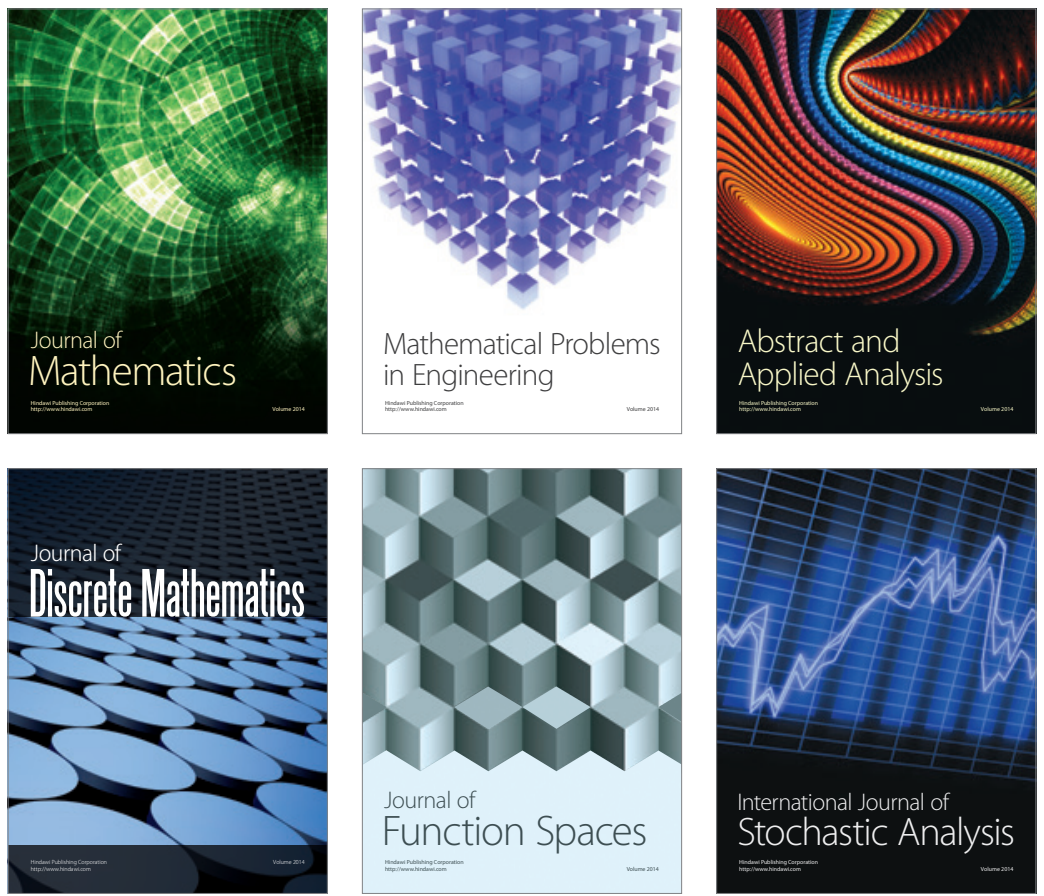

Journal of

Function Spaces

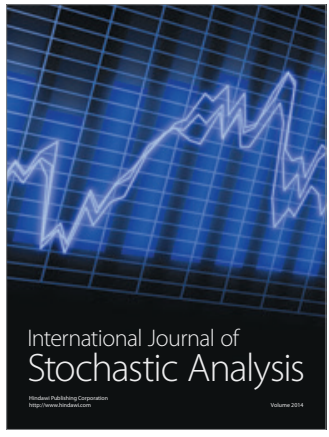

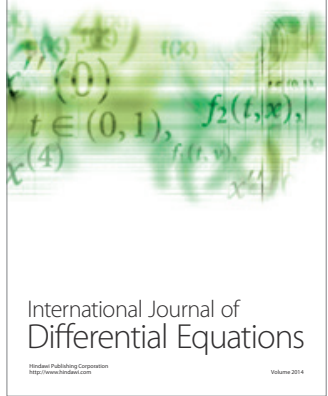
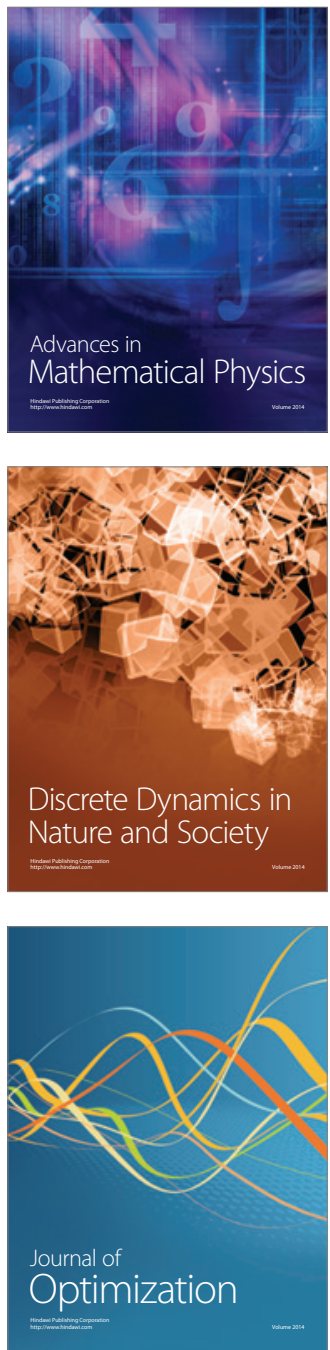